\title{
A combinatorial realization of Schur-Weyl duality via crystal graphs and dual equivalence graphs
}

\author{
Sami H. Assaf ${ }^{\dagger}$ \\ Department of Mathematics \\ University of Pennsylvania \\ Philadelphia, PA 19104-6395
}

\begin{abstract}
For any polynomial representation of the special linear group, the nodes of the corresponding crystal may be indexed by semi-standard Young tableaux. Under certain conditions, the standard Young tableaux occur, and do so with weight 0 . Standard Young tableaux also parametrize the vertices of dual equivalence graphs. Motivated by the underlying representation theory, in this paper, we explain this connection by giving a combinatorial manifestation of Schur-Weyl duality. In particular, we put a dual equivalence graph structure on the 0-weight space of certain crystal graphs, producing edges combinatorially from the crystal edges. The construction can be expressed in terms of the local characterizations given by Stembridge for crystal graphs and the author for dual equivalence graphs.
\end{abstract}

Keywords: Schur-Weyl duality, zero weight spaces, crystal graphs, dual equivalence graphs

\section{Introduction}

Schur-Weyl duality [Wey39] is a powerful tool in the study of irreducible representations of the classical groups. The set-up is as follows. Let $G$ be a complex, reductive Lie group, and let $V$ be an irreducible finite-dimensional complex representation of $G$. Let $V^{0}$ be the space of vectors of weight 0 , i.e. vectors which are fixed by a maximal torus. Then the Weyl group $W$ acts naturally on $V^{0}$. The case when $G=\mathrm{SL}_{n}$, and so then the Weyl group is isomorphic to $\mathcal{S}_{n}$, was studied by Gutkin [Gut73] who showed that if $V$ is an irreducible component of the $n$th tensor power of the standard $n$-dimensional representation of $\mathrm{SL}_{n}$, then the $\mathcal{S}_{n}$-module $V^{0}$ is irreducible and all irreducible $\mathcal{S}_{n}$-modules occur in this way. This was also observed by Kostant [Kos76]. Though there are descriptions of the Weyl group action on 0-weight spaces for $\mathrm{SL}_{n}$, there is no general rule for how to decompose the action into irreducible representations of $\mathcal{S}_{n}$. This paper proposes a new approach to this problem by combinatorializing the representations using crystal graphs [Kas90, Kas91] for representations of $\mathrm{SL}_{n}$ and dual equivalence graphs [Ass07] for representations of $\mathcal{S}_{n}$.

\footnotetext{
${ }^{\dagger}$ Work supported in part by NSF Mathematical Sciences Postdoctoral Research Fellowship DMS-0703567. 
Kashiwara introduced in [Kas90, Kas91] the notion of crystal bases in his study of the representation theory of quantized universal enveloping algebras at $q=0$. The theory of canonical bases, developed independently by Lusztig [Lus90a, Lus90b], studies the same problem from a different viewpoint, though for the purposes of this paper, we focus solely on the crystal approach. A crystal graph is a directed, colored graph with vertex set given by the crystal basis and directed edges given by deformations of the Chevalley generators $e_{i}$ and $f_{i}$. The combinatorial structure of crystal graphs encodes important information for studying the representations; for instance, knowing the crystal immediately gives a formula for the character as well as tensor product and branching rules for the corresponding representation. For the quantum group $U_{q}\left(\mathfrak{s l}_{n}\right)$, the crystal basis can be indexed by semi-standard Young tableaux, and there is an explicit combinatorial construction of the crystal graph on tableaux [KN94, Lit95].

Dual equivalence graphs were first introduced in [Ass07] as a combinatorial tool for studying functions expressed in terms of quasi-symmetric functions. In particular, in [Ass07] they are used to give a purely combinatorial proof of symmetry and Schur positivity of Lascoux-Leclerc-Thibon polynomials and Macdonald polynomials. The original motivation for these graphs, based on ideas of Haiman [Hai05], was to mimic existing crystal-theoretic proofs used to establish positivity results for functions expressed in terms of monomials. Given this, a natural problem which this paper addresses is to explore the connections between crystal graphs and dual equivalence graphs. Combinatorially, the vertices of a dual equivalence graph are standard Young tableaux of a given shape, which also index a basis for irreducible representations of $\mathcal{S}_{n}$. When $\lambda$ is a partition of $n$, these also index the 0 -weight nodes in the crystal graph of the irreducible $\mathrm{SL}_{n}$ module with highest weight $\lambda$. From this observation we develop a correspondence between crystals of irreducible representations of $\mathrm{SL}_{n}$ of degree $n$ and dual equivalence graphs.

This paper is organized as follows. Section 2 contains an exposition of type A crystal graphs. We begin by defining the combinatorial structure on semi-standard Young tableaux, and then present Stembridge's local characterization for crystals [Ste03] which will be essential for establishing our main result. Section 3 gives the analogous exposition for dual equivalence graphs, beginning with the combinatorial description in terms of standard Young tableaux before presenting the local characterization from [Ass07]. The correspondence is laid out in Section 4, where we give two separate proofs of the main result using the tableaux descriptions (Theorem 4.2) and the local characterizations (Theorem 4.3). Finally, in Section 5, we lay out how these methods may be extended to any irreducible representation of $\mathrm{SL}_{n}$ with a nontrivial 0 -weight space, and we also suggest how this approach may be used to define dual equivalence graphs for other types.

\section{Crystal graphs}

In order to construct the crystal graph of an irreducible representation of $\mathrm{SL}_{n}$, we must define Kashiwara's crystal operators in this setting. We depart somewhat from the original descriptions developed independently by Kashiwara and Nakashima [KN94] and Littelmann [Lit95] in favor of the presentation of the Littlewood-Richardson rule in [Mac95].

For a word $w$ of length $n$, i.e. $w \in \mathbb{N}^{n}$, define $m_{i}(w, r)$ to be the number of $i$ 's occurring in $w_{r} w_{r+1} \cdots w_{n}$ minus the number of $i-1$ 's occurring in $w_{r} w_{r+1} \cdots w_{n}$. Then let $m_{i}(w)$ be the maximum $m_{i}(w, r)$ which occurs for any $r=1, \ldots, n$. Observe that if $m_{i}(w)>0$ and $w_{p}$ is the rightmost occurrence of this maximum, i.e. $m_{i}(w, p)=m_{i}(w)$ and $m_{i}(w, q)<m_{i}(w)$ for $q>p$, then $w_{p}=i$. The result of applying the crystal operator $\tilde{e}_{i}$ to a semi-standard tableau $T$ may be described as follows. Let $w_{T}$ be the reading word for $T$. Then if $m_{i}\left(w_{T}\right) \leq 0, \tilde{e}_{i}(T)=0$; otherwise $\tilde{e}_{i}(T)$ is the result of 
changing the $i$ which is the rightmost occurrence of $m_{i}\left(w_{T}\right)$ to an $i-1$. As $\tilde{e}_{i}$ is invertible when nonzero, define $\tilde{f}_{i}$ by requiring $\tilde{e}_{i}$ and $\tilde{f}_{i}$ to be inverses of one another whenever the image is not 0 .

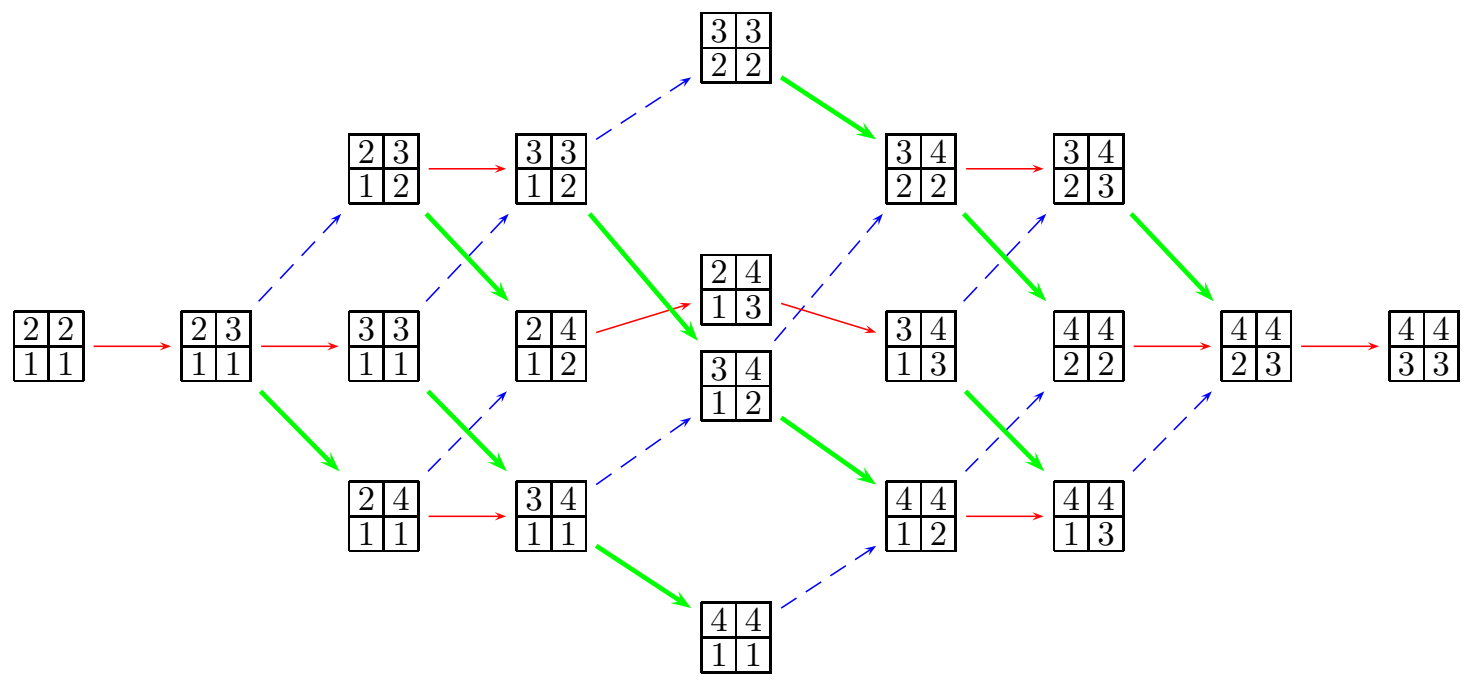

Fig. 1: The crystal graph $\mathcal{X}_{(2,2)}^{4}$, with edges $\tilde{f}_{1} \nearrow, \tilde{f}_{2} \rightarrow, \tilde{f}_{3} \backslash$.

For a partition $\lambda$ with at most $n$ rows, the crystal graph $\mathcal{X}_{\lambda}^{n}$ is the directed, colored graph on semistandard tableaux of shape $\lambda$ with entries from $[n]$ defined as follows. For $i=1, \ldots, n-1$, if $m_{i}\left(w_{T}\right)>0$, define a directed $i$-edge from $\tilde{e}_{i}(T)$ to $T$. For example, Figure 1 gives the crystal graph $\mathcal{X}_{(2,2)}^{4}$.

In [Ste03], Stembridge gives a local characterization of crystal graphs which arise from representations for simply-laced types. In order to define Stembridge's axioms, we must first introduce some notation associated with a directed, colored graph. Say that $\mathcal{X}$ has degree $n$ if the largest color for an edge is $n-1$.

We have simplified things from the presentation in [Ste03] since we are considering only the type A case. The notations below may not be well-defined in general, but will be in the contexts in which they are used, i.e. for graphs satisfying axioms $\mathrm{P} 1$ and $\mathrm{P} 2$ below.

If $x \stackrel{i}{\longleftarrow} y$ (resp. $x \stackrel{i}{\longrightarrow} z$ ), write $y=E_{i} x$ (resp. $z=F_{i} x$ ). The $i$-string through $x$ is the maximal path

$$
F_{i}^{-d} x \stackrel{i}{\longrightarrow} \cdots \stackrel{i}{\longrightarrow} F_{i}^{-1} x \stackrel{i}{\longrightarrow} x \stackrel{i}{\longrightarrow} F_{i} x \stackrel{i}{\longrightarrow} \cdots \stackrel{i}{\longrightarrow} F_{i}^{r} x .
$$

In this case we write $\delta(x, i)=-d$ and $\varepsilon(x, i)=r$. Finally, we have the following differences whenever $E_{i}, F_{i}$ is defined at $x$.

$$
\begin{aligned}
\Delta_{i} \delta(x, j) & =\delta\left(E_{i} x, j\right)-\delta(x, j), & \nabla_{i} \delta(x, j) & =\delta(x, j)-\delta\left(F_{i} x, j\right), \\
\Delta_{i} \varepsilon(x, j) & =\varepsilon\left(E_{i} x, j\right)-\varepsilon(x, j), & \nabla_{i} \varepsilon(x, j) & =\varepsilon(x, j)-\varepsilon\left(F_{i} x, j\right) .
\end{aligned}
$$


Definition 2.1 ([Ste03]) A directed, colored graph $\mathcal{X}$ is regular if the following hold:

(P1) all monochromatic directed paths have finite length;

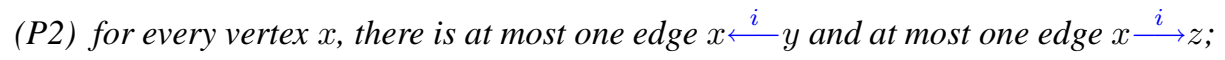

(P3) assuming $E_{i} x$ is defined, $\Delta_{i} \delta(x, j)+\Delta_{i} \varepsilon(x, j)=\left\{\begin{aligned} 2 & \text { if } j=i \\ -1 & \text { if } j=i \pm 1 \\ 0 & \text { if }|i-j| \geq 2\end{aligned}\right.$;

(P4) assuming $E_{i} x$ is defined, $\Delta_{i} \delta(x, j), \Delta_{i} \varepsilon(x, j) \leq 0$ for $j \neq i$;

(P5) $\Delta_{i} \delta(x, j)=0 \Rightarrow E_{i} E_{j} x=E_{j} E_{i} x=y$ and $\nabla_{j} \varepsilon(y, i)=0$;

$\nabla_{i} \varepsilon(x, j)=0 \Rightarrow F_{i} F_{j} x=F_{j} F_{i} x=y$ and $\Delta_{j} \delta(y, i)=0 ;$

(P6) $\Delta_{i} \delta(x, j)=\Delta_{j} \delta(x, i)=-1 \Rightarrow E_{i} E_{j}^{2} E_{i} x=E_{j} E_{i}^{2} E_{j} x=y$ and $\nabla_{i} \varepsilon(y, j)=\nabla_{j} \varepsilon(y, i)=-1$; $\nabla_{i} \varepsilon(x, j)=\nabla_{j} \varepsilon(x, i)=-1 \Rightarrow F_{i} F_{j}^{2} F_{i} x=F_{j} F_{i}^{2} F_{j} x=y$ and $\Delta_{i} \delta(y, j)=\Delta_{j} \delta(y, i)=-1$.

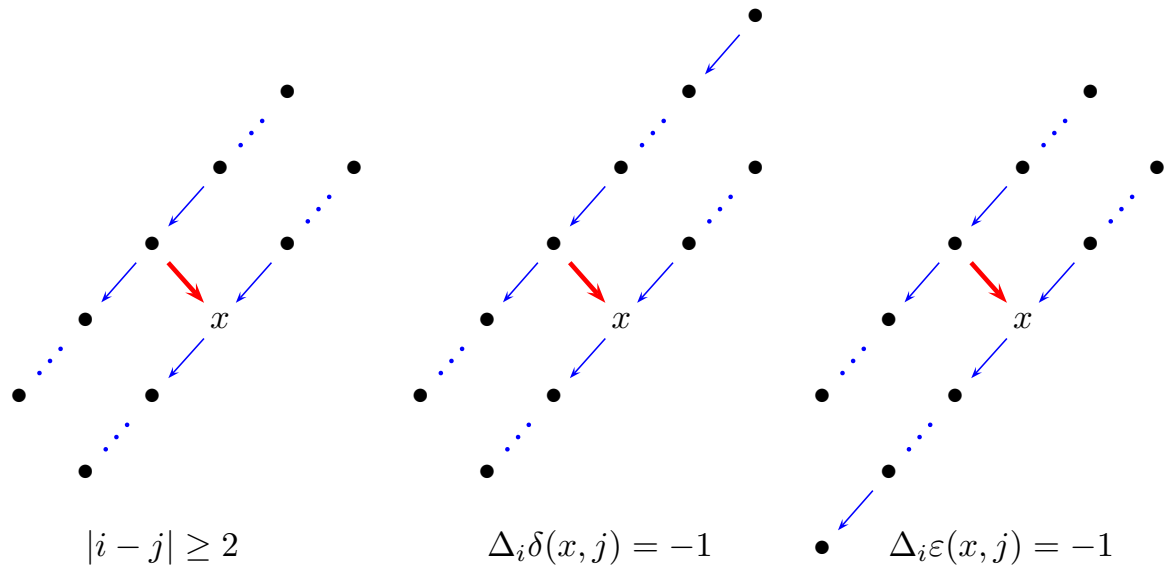

Fig. 2: An illustration of axioms $\mathrm{P} 3$ and $\mathrm{P} 4$, where $F_{j} \swarrow, F_{i} \searrow$.

Axioms P3 and P4 dictate how the maximal length of a $j$-string differs between $x$ and $E_{i} x$; see Figure 2. When $|i-j| \geq 2$, there is no change, but when $j=i \pm 1$, the length must change by 1 . Axioms P5 and P6 give information about how edges with different labels interact; see Figure 3. When $|i-j| \geq 2$, the conditions of P5 will always be satisfied, though when $j=i \pm 1$, either P5 or P6 could hold.

The following theorem shows that regular graphs corresponds precisely to the crystal graphs of representations in type A. This result allows us to combinatorialize the problem of studying representations of $\mathrm{SL}_{n}$ by instead studying regular graphs of degree $n$. 


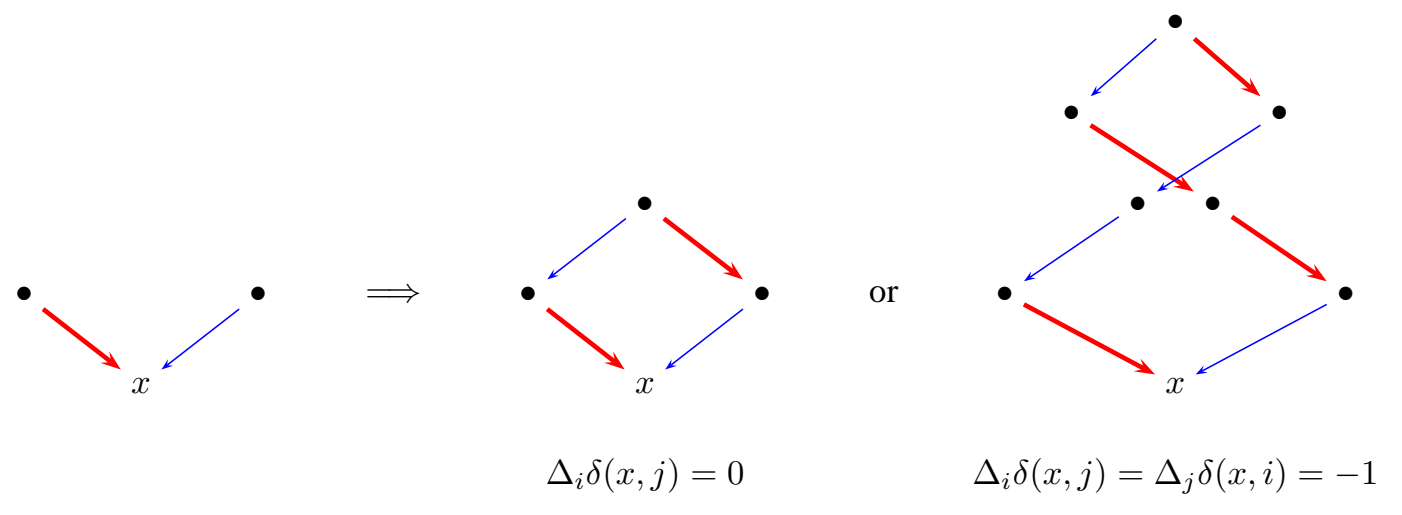

Fig. 3: An illustration of axioms $\mathrm{P} 5$ and $\mathrm{P} 6$, where $F_{j} \swarrow, F_{i} \searrow$.

Theorem 2.2 ([Ste03]) Each $\mathcal{X}_{\lambda}^{n}$ is a regular graph, and every connected component of a regular graph is isomorphic to $\mathcal{X}_{\lambda}^{n}$ for some $\lambda, n$.

The theorem is proved by showing that Littelmann's path operators [Lit94] generate regular graphs, since the Path Model is known to generate the $\mathcal{X}_{\lambda}^{n}$. As remarked in [Ste03], it would be nice to have a graph-theoretic proof of Theorem 2.2 which bypasses the Path Model.

\section{Dual equivalence graphs}

Since the characters of irreducible representations of $\mathrm{SL}_{n}$ are Schur polynomials which form a basis for symmetric polynomials, crystal graphs can be used to prove that certain polynomials expressed in terms of monomials are symmetric and Schur positive. Motivated by this approach, Haiman [Hai05] suggested defining a graph structure on standard tableaux using the dual equivalence relation as a means of establishing the symmetry and Schur positivity of polynomials expressed in terms of quasi-symmetric functions. The result of this idea is the theory of dual equivalence graphs developed in [Ass07].

Following Haiman's idea, we use the dual equivalence relation to construct a graph whose vertices are given by standard tableaux and whose connected components are indexed by partitions.

Definition 3.1 ([Hai92]) An elementary dual equivalence on three consecutive letters, say $i-1, i, i+1$, of a permutation is given by switching the outer two letters whenever the middle letter is not $i$ :

$$
\cdots i \cdots i \pm 1 \cdots i \mp 1 \cdots \equiv^{*} \cdots i \mp 1 \cdots i \pm 1 \cdots i \cdots
$$

The standard dual equivalence graph associated to $\lambda$, denoted $\mathcal{G}_{\lambda}$, is the vertex-signed, edge-colored graph constructed in the following way. The vertices are given by all standard Young tableaux of shape $\lambda$. Two tableaux $T$ and $U$ are connected by an $i$-colored whenever their reading words, $w_{T}$ and $w_{U}$, differ by an elementary dual equivalence for $i-1, i, i+1$. To each vertex $T$ we associate the signature $\sigma(T)$ which indicates the descent set of the tableau:

$$
\sigma(T)_{i}=\left\{\begin{array}{ll}
+1 & \text { if } i \text { appears to the left of } i+1 \text { in } w_{T} \\
-1 & \text { if } i+1 \text { appears to the left of } i \text { in } w_{T}
\end{array} .\right.
$$


As the original motivation for these graphs was to prove Schur positivity, the role of the signatures is to index which quasi-symmetric function should be associated to each vertex.

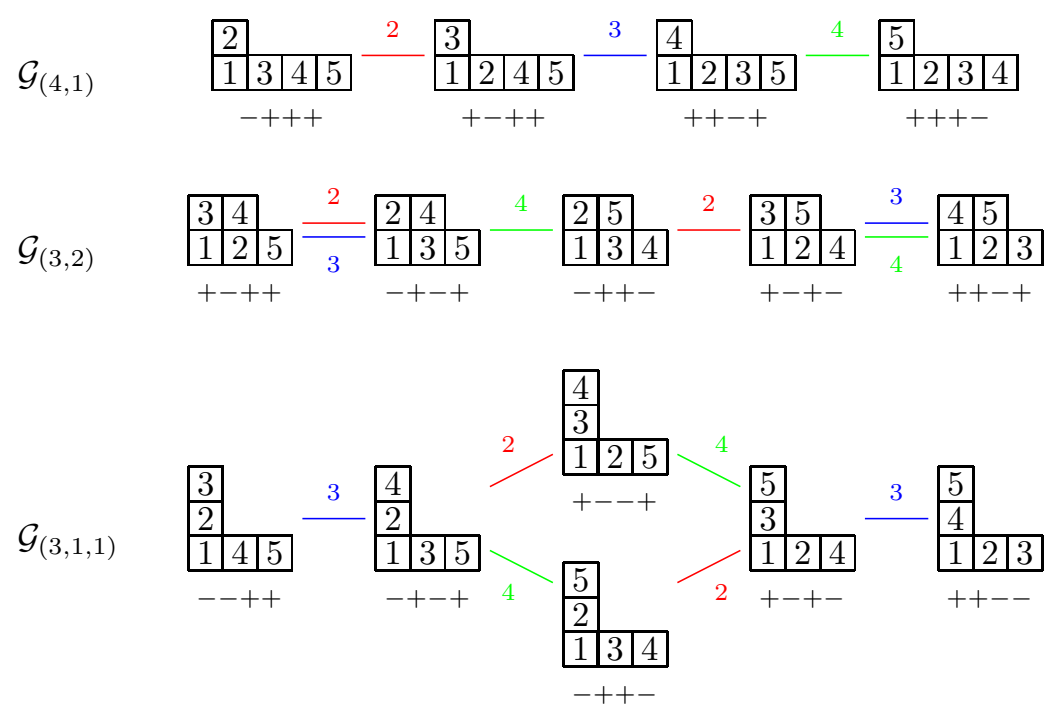

Fig. 4: The standard dual equivalence graphs $\mathcal{G}_{(4,1)}, \mathcal{G}_{(3,2)}$ and $\mathcal{G}_{(3,1,1)}$.

In contrast with $\mathcal{X}_{\lambda}^{n}$, the bound on the edge colors (equivalently, on the entries of the tableaux) for $\mathcal{G}_{\lambda}$ is implicit. More precisely, if $\lambda$ is a partition of $m$, then $\mathcal{G}_{\lambda}$ has degree $m$, meaning that the largest edge color is $m-1$.

It follows from results in [Hai92] that each $\mathcal{G}_{\lambda}$ is connected. It is easy to see from the construction that $\mathcal{G}_{\lambda^{\prime}}$ can be obtained from $\mathcal{G}_{\lambda}$ by conjugating each vertex and multiplying each entry of the signature by -1 . Several other nice properties of these graphs are given [Ass07], including the fact that the $\mathcal{G}_{\lambda}$ are pairwise non-isomorphic and have no nontrivial automorphisms.

Motivated by the local characterization of crystal graphs $\mathcal{X}_{\lambda}^{n}$, we next present a local characterization of the graphs $\mathcal{G}_{\lambda}$. We will abuse notation by simultaneously referring to $D_{i}$ as the collection of $i$-colored edges as well as an involution on vertices which have an $i$-edge, i.e. we write $D_{i}(v)=w$ whenever $\{v, w\} \in D_{i}$. This is justified by the first axiom.

Definition 3.2 ([Ass07]) A signed, colored graph $\mathcal{G}=(V, \sigma, D)$ is a dual equivalence graph if the following hold:

(axl) For $v \in V$ and $i>1, \sigma(v)_{i-1}=-\sigma(v)_{i}$ if and only if there exists $w \in V$ such that $\{v, w\} \in D_{i}$. Moreover, $w$ is unique when it exists.

(ax2) Whenever $\{v, w\} \in D_{i}$,

$$
\begin{aligned}
& \sigma(v)_{j}=-\sigma(w)_{j} \quad \text { for } j=i-1, i \text {; } \\
& \sigma(v)_{h}=\sigma(w)_{h} \quad \text { for } h<i-2 \text { and } h>i+1 \text {. }
\end{aligned}
$$


(ax3) Whenever $\{v, w\} \in D_{i}$,

$$
\begin{aligned}
& \text { if } \sigma(v)_{i-2}=-\sigma(w)_{i-2}, \quad \text { then } \sigma(v)_{i-2}=-\sigma(v)_{i-1} \\
& \text { if } \sigma(v)_{i+1}=-\sigma(w)_{i+1}, \quad \text { then } \sigma(v)_{i+1}=-\sigma(v)_{i} .
\end{aligned}
$$

(ax4) For $i>3$, every non-trivial connected component of $\left(V, \sigma, D_{i-2} \cup D_{i-1} \cup D_{i}\right)$ is depicted below and there is a path containing at most one $D_{i}$ edge between any two vertices of $\left(V, \sigma, D_{2} \cup \cdots \cup D_{i}\right)$.

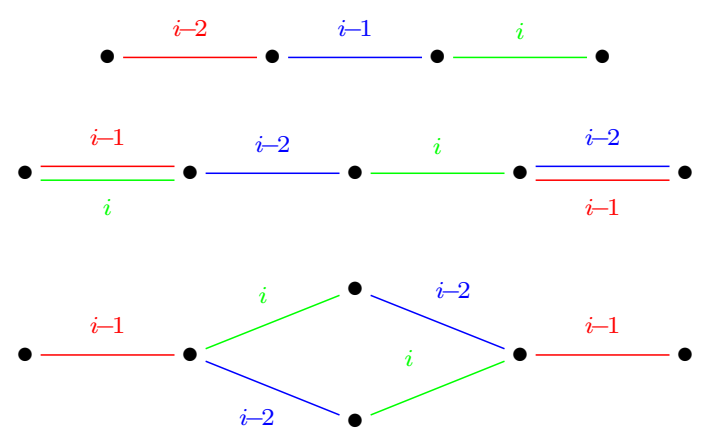

(ax5) Whenever $|i-j| \geq 3,\{v, u\} \in D_{i}$ and $\{u, w\} \in D_{j}$, there exists $x \in V$ such that $\{v, x\} \in D_{j}$ and $\{x, w\} \in D_{i}$.

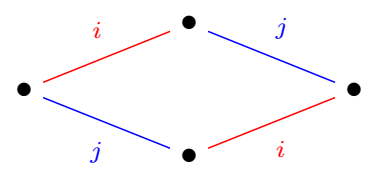

When $n=4$, we stipulate the following in place of axiom 4: For $i>2$, every non-trivial connected component of the sub-graph $\left(V, \sigma, D_{i-1} \cup D_{i}\right)$ is one of the following:

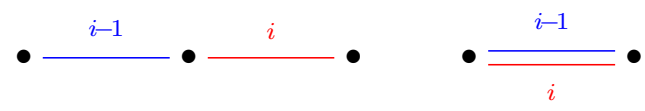

Next we have the analog of Theorem 2.2 for dual equivalence graphs. The proof of Theorem 3.3 is by a purely combinatorial, graph-theoretic argument.

Theorem 3.3 ([Ass07]) Each $\mathcal{G}_{\lambda}$ is a dual equivalence graph, and every connected component of a dual equivalence graph is isomorphic to $\mathcal{G}_{\lambda}$ for a unique partition $\lambda$.

\section{From crystal graphs to dual equivalence graphs}

Since standard tableaux are special cases of semi-standard tableaux, and since the construction of $\mathcal{G}_{\lambda}$ was motivated by $\mathcal{X}_{\lambda}^{n}$, we would like to explore the connection between regular graphs and dual equivalence graphs. Since semi-standard tableaux index a basis for an irreducible representation of $\mathrm{SL}_{n}$ so that the content of the tableau corresponds to the weight of the basis vector, those basis vectors corresponding 
to standard tableaux index the 0 -weight space of the representations of dimension $n$. Therefore we may think of dual equivalence graphs as living in the 0-weight space of crystal graphs.

In general, the Weyl group acts naturally on the 0-weight space of a representation. In the case of $\mathrm{SL}_{n}$, the Weyl group is isomorphic to $\mathcal{S}_{n}$. Since standard tableaux index the basis for an irreducible representation of $\mathcal{S}_{n}$, we would like to use dual equivalence graphs to combinatorialize the study of representations of $\mathcal{S}_{n}$ in much the same way that crystal graphs combinatorialize the study of representations of $\mathrm{SL}_{n}$. This is the motivation behind the following correspondence. In the interest of space, the longer proofs have been condensed by omitting the more mechanical details.

Combinatorially, the 0 -weight space of a regular graph $\mathcal{X}$ consists of all vertices which lie at the center of each $i$-string; i.e. $\varepsilon(x, i)=-\delta(x, i)$ for each $i$. Given a directed, colored graph $\mathcal{X}$, define a signed, colored graph $\mathcal{G}(\mathcal{X})=(V, \sigma, D)$ by

$$
\begin{aligned}
V & =\{x \in \mathcal{X} \mid \varepsilon(x, i)=-\delta(x, i)=0 \text { or } 1 \forall i,\}, \\
\sigma(x)_{i} & =\left\{\begin{array}{ll}
+1 & \text { if } \varepsilon(x, i)=1 \\
-1 & \text { if } \varepsilon(x, i)=0
\end{array},\right. \\
D_{i}(x) & =\left\{\begin{array}{ll}
F_{i-1} F_{i} E_{i-1} E_{i} x & \text { if } \varepsilon(x, i)=1 \text { and } \varepsilon(x, i-1)=0 \\
F_{i} F_{i-1} E_{i} E_{i-1} x & \text { if } \varepsilon(x, i)=0 \text { and } \varepsilon(x, i-1)=1
\end{array} .\right.
\end{aligned}
$$

An $i$-edge from $x$, or equivalently $D_{i}(x)$, is not defined if neither condition of equation (4.3) holds.

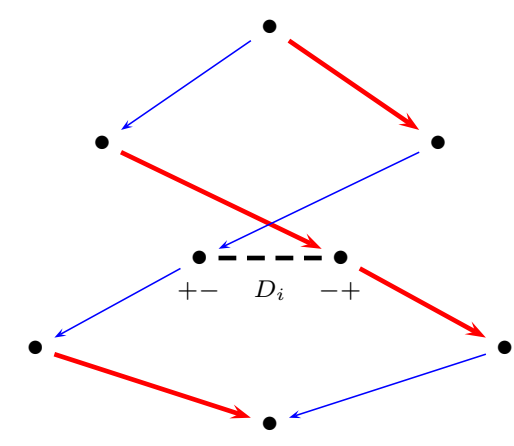

Fig. 5: Constructing $\mathcal{G}(\mathcal{X})$ from $\mathcal{X}$; here we have $F_{i-1 \swarrow} \swarrow, F_{i} \searrow$.

Proposition 4.1 If $\mathcal{X}$ is a regular graph, then $\mathcal{G}(\mathcal{X})$ is well-defined.

Proof: It suffices to show that $D_{i}$ is well-defined. Note that if $\delta(x, i)=-1$, then $E_{i} x$ is nonzero. If $\delta(x, i-1)=0$, then by axiom P3 we must have $\delta\left(E_{i} x, i-1\right)+\varepsilon\left(E_{i} x, i-1\right)=-1$. The constraints of axiom $\mathrm{P} 4$ force $\varepsilon\left(E_{i} x, i-1\right)=0$, and so we conclude that $\delta\left(E_{i} x, i-1\right)=-1$. Therefore $E_{i-1} E_{i} x$ is nonzero. Continuing thus, with liberal use of axioms $\mathrm{P} 3$ and $\mathrm{P} 4$, we eventually conclude that $F_{i-1} F_{i} E_{i-1} E_{i} x$ is nonzero. The analogous argument holds with the reverse assumptions for $\delta(x, i-1), \delta(x, i)$. 
Below we give two separate proofs that if $\mathcal{X}$ is a regular graph with 0 -weight space given by $V$, then $\mathcal{G}(\mathcal{X})$ is a dual equivalence graph. The first proof utilizes the graph structures on tableaux, and the second uses the local characterizations. Note that the condition that $V$ give the 0 -weight space is highly restrictive.

In order for $\mathcal{X}_{\lambda}^{n}$ to have a 0 -weight space, $\lambda$ must be a partition of $k n$ into at most $n$ parts. We first treat the case for $\mathcal{X}_{\lambda}^{n}$ when $k=1$, i.e. $\lambda$ is a partition of $n$.

Theorem 4.2 For every partition $\lambda$ of $n$, we have $\mathcal{G}\left(\mathcal{X}_{\lambda}^{n}\right)=\mathcal{G}_{\lambda}$.

Proof: In this case we may identify $V$ with $\operatorname{SYT}(\lambda)$. Let $w_{T}$ be the reading word for a tableau $T$. For $T \in \operatorname{SYT}(\lambda)$, note that $\varepsilon(T, i)=0$ if and only if $i+1$ occurs to the left of $i$ in $w_{T}$. Therefore equations 3.1 and 4.2 correspond. In particular, $T$ will have an $i$-edge in $\mathcal{G}_{\lambda}$ precisely when equation (4.3) is defined.

Suppose $D_{i}(T)$ is defined, and so $T$ admits an elementary dual equivalence for $i-1, i, i+1$. By symmetry, we may assume $i$ lies to the left of $i \pm 1$ in $w_{T}$. If $\varepsilon(T, i)=1$, then ignoring all letters other than $i-1, i, i+1$ and regarding $\tilde{e}_{j}, \tilde{f}_{j}(j=i-1, i)$ as operators on reading words, we have

$$
\begin{aligned}
& \tilde{f}_{i-1} \tilde{f}_{i} \tilde{e}_{i-1} \tilde{e}_{i} w_{T}=\tilde{f}_{i-1} \tilde{f}_{i} \tilde{e}_{i-1} \tilde{e}_{i} \quad \cdots \quad i \quad \cdots i-1 \cdots i+1 \cdots \\
& =\tilde{f}_{i-1} \tilde{f}_{i} \tilde{e}_{i-1} \quad \cdots \quad i \quad \cdots i-1 \cdots \quad i \quad \cdots \\
& =\quad \begin{array}{rllll}
\tilde{f}_{i-1} \tilde{f}_{i} & \cdots & i & \cdots i-1 \cdots i-1 \cdots
\end{array} \\
& =\quad \tilde{f}_{i-1} \quad \cdots i+1 \cdots i-1 \cdots i-1 \cdots \\
& =\quad \cdots i+1 \cdots i-1 \cdots i \ldots
\end{aligned}
$$

Therefore equation (4.3) corresponds to the elementary dual equivalences. The case when $\varepsilon(T, i-1)=1$ is similar.

Going back to the representation theory of $\mathrm{SL}_{n}$ and $\mathcal{S}_{n}$, Theorem 4.2 reflects the fact that for $\lambda$ a partition of $n$, the action of $\mathcal{S}_{n}$ on the 0 -weight space of the irreducible representation of $\mathrm{SL}_{n}$ corresponding to $\lambda$ is given by the Specht module $S^{\lambda}$.

The following result generalizes Theorem 4.2. Here we present a self-contained proof based solely on the local characterizations of crystal graphs and dual equivalence graphs without appealing to Theorems 2.2 and 3.3 and without reference to tableaux.

Theorem 4.3 If $\mathcal{X}$ is a regular with 0 -weight space given by $V$ (see equation (4.1)), then $\mathcal{G}(\mathcal{X})$ is a dual equivalence graph.

Proof: Comparing equation (4.2) with the conditions in equation (4.3) dictating when $D_{i}$ is defined shows that $\sigma(x)_{i-1}=-\sigma(x)_{i}$ if and only if $D_{i}(x)$ is defined. Figure 5 makes evident the fact that $\sigma(x)_{j}=-\sigma\left(D_{i}(x)\right)_{j}$ for $j=i-1, i$, though this can also be seen directly from the proof that $D_{i}$ is well-defined. Therefore we have established dual equivalence axioms 1 and 2.

Axiom 3 can be demonstrated by showing that if $\varepsilon(x, i-2) \neq \varepsilon\left(D_{i}(x), i-2\right)$, then $D_{i}(x)=D_{i-1}(x)$. To do this, we show that the crystal graph operators going from $x$ to $D_{i}(x)$ exactly mirror the situation in $\mathcal{X}_{(2,2)}^{4}$ for the two standard tableaux of shape $(2,2)$; see Figure 1.

By crystal axioms P3, P4 and P5, whenever $|a-b| \geq 2$, we have $E_{a} E_{b}=E_{b} E_{a}, F_{a} E_{b}=E_{b} F_{a}$ and $F_{a} F_{b}=F_{b} F_{a}$. Dual equivalence axiom 5 is equivalent to showing that if $D_{i}$ and $D_{j}$ are both defined at $x$ for some $|i-j| \geq 3$, then $D_{i} D_{j}(x)=D_{j} D_{i}(x)$. Therefore the identities above for crystal operators establish dual equivalence axiom 5 . 


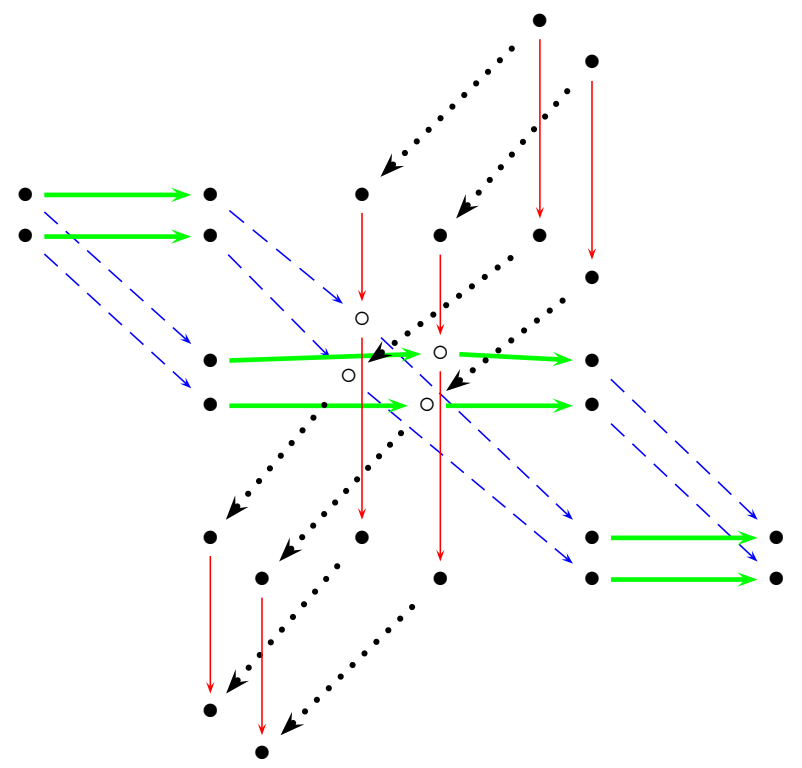

Fig. 6: An illustration of the commutativity which establishes dual equivalence axiom 4; the 0 -weight vertices are ○'s and crystal operators are $F_{i-3} \searrow, F_{i-2} \rightarrow, F_{i-1} \swarrow, F_{i} \downarrow$.

The most difficult step is to establish axiom 4. For this, note that it is sufficient to show that if $D_{i-2}, D_{i-1}, D_{i}$ are all defined at $x$ and are all distinct (i.e. $x$ does not have a double edge), then $D_{i-2} D_{i}(x)=$ $D_{i} D_{i-2}(x)$. By symmetry we may assume $\varepsilon(x, i-3)=1=\varepsilon(x, i-1)$ and $\varepsilon(x, i-2)=0=\varepsilon(x, i)$. Then the first part of axiom 4 is equivalent to establishing the following identity, illustrated in Figure 6.

$$
\left(F_{i} F_{i-1} E_{i} E_{i-1}\right)\left(F_{i-2} F_{i-3} E_{i-2} E_{i-3}\right) x=\left(F_{i-2} F_{i-3} E_{i-2} E_{i-3}\right)\left(F_{i} F_{i-1} E_{i} E_{i-1}\right) x
$$

The key to establishing this relation is to use the commutativity relations for crystal operators used to prove dual equivalence axiom 5, as well as the additional relation that both $E_{i-1}$ and $F_{i-1}$ commute with the sequence $F_{i-2} F_{i-3} E_{i-2}$.

For the second part of axiom 4, we first show that if $\mathcal{X}$ is connected, then so is $\mathcal{G}(\mathcal{X})$. In particular, we show that any two vertices of $V$ may be connected by a path consisting of sequences as in equation (4.3). Furthermore, for any two vertices we may choose a path containing at most one sequence using the maximal edge color. Now axiom 4 follows.

Corollary 4.4 Let $\lambda=\left(\mu_{1}+k, \mu_{2}+k, \ldots, \mu_{n}+k\right)$ for some partition $\mu$ of $n$ and some non-negative integer $k$. Then $\mathcal{G}\left(\mathcal{X}_{\lambda}^{n}\right)=\mathcal{G}_{\mu}$.

The partition $\lambda$ is the result of adding $k$ columns of height $n$ to $\mu$, and clearly has 0 -weight space given by $V$. Such a graph corresponds to the irreducible representation of $\mathrm{SL}_{n}$ indexed by $\mu$, since $\mathrm{SL}_{n}$ cannot discriminate between the irreducible representations indexed by $\lambda$ and $\mu$. Therefore Corollary 4.4 shows 
that the corresponding dual equivalence graph decomposes (albeit trivially) the action of the Weyl group on the 0 -weight space of the representation.

Remark 4.5 By introducing weights to the regular graphs, we can recover the dimension of a regular graph, which in the context of Theorem 2.2 gives the size of the indexing partition. Letting $k$ denote the ratio of the dimension of $\mathcal{X}$ to the degree of $\mathcal{X}$, modify the definition of $\sigma$ in equation (4.2) to be

$$
\sigma(x)_{i}=(-1)^{\varepsilon(x, i)+k} .
$$

Since multiplying the signatures of a dual equivalence graph entry-wise by -1 has the effect of conjugating the indexing partition, under this definition Corollary 4.4 must read

$$
\mathcal{G}\left(\mathcal{X}_{\lambda}^{n}\right)= \begin{cases}\mathcal{G}_{\mu} & \text { if } k \text { is odd } \\ \mathcal{G}_{\mu^{\prime}} & \text { if } k \text { is even }\end{cases}
$$

Representation theoretically, this corresponds to tensoring with the determinant representation in $\mathrm{GL}_{n}$, which has the effect of conjugating the shape. So indeed, the dual equivalence graph on the 0-weight space of $\mathcal{X}_{\lambda}^{n}$ decomposes the action of $\mathcal{S}_{n}$ on the 0 -weight space of the corresponding representation of $\mathrm{GL}_{n}$ with this minor modification of signatures.

\section{Extensions}

The ultimate goal is to extend the construction of $\mathcal{G}(\mathcal{X})$ for any regular graph $\mathcal{X}$ which has a 0 -weight space. The characterization of $V$ in equation (4.1) would become

$$
V=\{x \in \mathcal{X} \mid \varepsilon(x, i)=-\delta(x, i) \forall i\}
$$

In many cases, the 0-weight graphs obtained from regular graphs in a similar manner can be viewed as D graphs, which are generalizations of dual equivalence graphs; see [Ass07]. Each D graph can be transformed into a disjoint union of dual equivalence graphs, so the hope is that each 0-weight graph may be realized as a D graph which decomposes into dual equivalence graphs in the correct way. If successful, this would provide a combinatorial way to study the representations of Weyl groups on 0-weight spaces, at least in type A.

A further direction is to use the correspondence between type A crystal graphs and dual equivalence graphs to define dual equivalence graphs in other types. For the simply-laced types at least, this can be done by the same constructions using Stembridge's axioms. Using the combinatorial description of crystal bases in terms of generalized Young tableaux [KN94, Lit95], we may define the standard graphs which are the analogs of $\mathcal{G}_{\lambda}$, and then use the local characterization of crystal graphs to obtain a local characterization for dual equivalence graphs.

\section{Acknowledgements}

The author is grateful to Mark Haiman for the idea to construct the standard dual equivalence graph $\mathcal{G}_{\lambda}$ and for suggesting investigating the connections with crystal graphs. The author also thanks Ian Grojnowski, Mark Haiman and Monica Vazirani for helpful conversations about crystal graphs and 0-weight spaces. 


\section{References}

[Ass07] S. Assaf, A combinatorial proof of LLT and Macdonald positivity, preprint, 2007.

[Gut73] E. A. Gutkin, Representations of the Weyl group in the space of vectors of zero weight, Uspehi Mat. Nauk 28 (1973), no. 5 (173), 237-238.

[Hai92] Mark D. Haiman, Dual equivalence with applications, including a conjecture of Proctor, Discrete Math., 99 (1992), no. 1-3, 79-113.

[Hai05] Mark Haiman, personal communication, 2005.

[Kas90] Masaki Kashiwara, Crystalizing the q-analogue of universal enveloping algebras, Comm. Math. Phys. 133 (1990), no. 2, 249-260.

[Kas91] M. Kashiwara, On crystal bases of the Q-analogue of universal enveloping algebras, Duke Math. J. 63 (1991), no. 2, 465-516.

[KN94] Masaki Kashiwara and Toshiki Nakashima, Crystal graphs for representations of the qanalogue of classical Lie algebras, J. Algebra 165 (1994), no. 2, 295-345.

[Kos76] Bertram Kostant, On Macdonald's $\eta$-function formula, the Laplacian and generalized exponents, Advances in Math. 20 (1976), no. 2, 179-212.

[Lit94] Peter Littelmann, A Littlewood-Richardson rule for symmetrizable Kac-Moody algebras, Invent. Math. 116 (1994), no. 1-3, 329-346.

[Lit95] Peter Littelmann, Crystal graphs and Young tableaux, J. Algebra 175 (1995), no. 1, 65-87.

[Lus90a] G. Lusztig, Canonical bases arising from quantized enveloping algebras, J. Amer. Math. Soc. 3 (1990), no. 2, 447-498.

[Lus90b] G. Lusztig, Canonical bases arising from quantized enveloping algebras. II, Progr. Theoret. Phys. Suppl. (1990), no. 102, 175-201 (1991), Common trends in mathematics and quantum field theories (Kyoto, 1990).

[Mac95] I. G. Macdonald, Symmetric functions and Hall polynomials, second ed., Oxford Mathematical Monographs, The Clarendon Press Oxford University Press, New York, 1995, With contributions by A. Zelevinsky, Oxford Science Publications.

[Ste03] John R. Stembridge, A local characterization of simply-laced crystals, Trans. Amer. Math. Soc. bf 355 (2003), no. 12, 4807-4823 (electronic).

[Wey39] Hermann Weyl, The Classical Groups. Their Invariants and Representations, Princeton University Press, Princeton, N.J., 1939. 